\title{
DETERMINATION OF THE INTERNAL RESISTANCE OF A HAMMER DRILL CHISEL
}

\author{
NikOLAY NikOLOV \\ Technical University - Sofia, Strength of Materials Department, Sofia, Bulgaria \\ e-mail:nyky@tu-sofia.bg \\ Petko Sinapov \\ Technical University - Sofia, Department of Mechanics, Sofia, Bulgaria \\ e-mail:p_sinapov@tu-sofia.bg
}

\begin{abstract}
The present work examines damped oscillations of a chisel, represented as a distributed parameters system. The system is discretized with the finite element method. Rayleigh's law is used for the modelling of the resistance. The internal resistance of the mechanical system has been determined by an experiment, then enshrined in the numerical model. Comparison and analysis of the results have been made.
\end{abstract}

Keywords: free damped oscillations, FEM, Rayleigh damping matrix, damping coefficients

\section{Introduction}

Precise determination of the internal resistance is a crucial point in the research on the distributed parameter dynamics of mechanical systems. Without knowing its value, it would be impossible to study numerically different processes, such as free damped or forced oscillations, nonlinear oscillations, shock occurrences and others. The Rayleigh law is most commonly used to describe the internal resistance

$$
\mathbf{C}=\alpha \mathbf{M}+\beta \mathbf{K}
$$

where $\mathbf{C}$ is the Rayleigh damping matrix, $\alpha$ is a coefficient taking into account the influence of the mass distribution on the resistance, $\beta$ is a coefficient accounting for the influence of the system elasticity on the resistance, while $\mathbf{M}$ and $\mathbf{K}$ are, respectively, mass and stiffness matrices. According to many researchers (Stelzmann et al., 2008), the coefficients $\alpha$ and $\beta$ are to be determined for each specific structure. It turns out that by varying these two coefficients, certain oscillation frequencies could be supressed, while others prevail. A number of studies have shown (Man and Corman, 1995) that the $\alpha$-damping affects low frequencies, and the $\beta$-damping affects high frequencies, as shown in Fig. 1. A great advantage when using the Rayleigh law is that after orthogonal transformation the system of $n$ differential equations, describing motion of the system, can be represented by $n$ independent equations (Chowdhury and Dasgupta, 2003), i.e. it can break the connection between the equations.

Different methods could be used for determining the $\alpha$ and $\beta$ (Craig and Kurdila, 2006; Alipour and Zareian, 2008; Adhikari, 2001). If $\omega_{1}^{*}$ and $\omega_{2}^{*}$ are the first two frequencies of the system damped oscillations, then for the most common method it is assumed that at both ends of the frequency range $\left[\omega_{1}^{*} ; \omega_{2}^{*}\right]$ the damping ratio $\xi$ has equal values (Fig. 1). When a mechanical system is dominated by free oscillations with frequencies within the mentioned range, the values of $\alpha$ and $\beta$ are determined by the equations

$$
\alpha=\xi \frac{2 \omega_{1}^{*} \omega_{2}^{*}}{\omega_{1}^{*}+\omega_{2}^{*}} \quad \beta=\xi \frac{2}{\omega_{1}^{*}+\omega_{2}^{*}}
$$




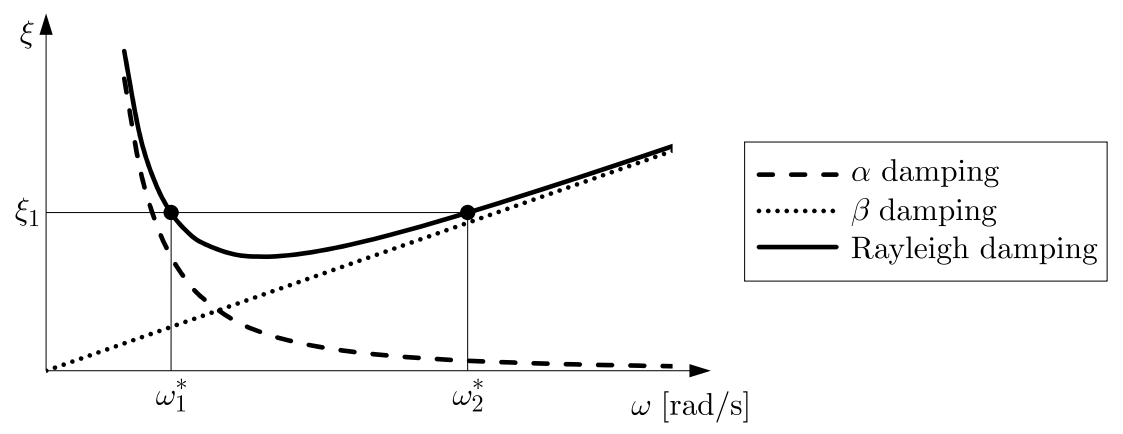

Fig. 1. Rayleigh damping

The damping ratio $\xi$ depends on the material and the studied structure. Information about the value of $\xi$ for steel can be found in the bibliography. For example, the following intervals from 0.02 to 0,08 ; from 0.02 to 0.03 and from 0.0008 to 0.0025 are given respectively in (Chowdhury and Dasgupta, 2003; Chopra, 1995; Dresig and Holzweissig, 2006). In (Mevada and Patel, 2016) $\xi$ was experimentally determined and the obtained value was 0.0069. It was found by Stevenson (1980) that $\xi$ depended on the dynamic values of the stresses and strains, and the value for steel was set to 0.01. In (Zare et al., 2011) $\xi$ has the value of 0.03 and in (Sangeetha et al., 2014) $\xi$ has the value of 0.2 . It appears that for each structure the resistance is strictly individual and it often depends even on the operation mode.

The purpose of the present work is to model damping oscillations of the mechanical system once the internal resistance has been experimentally determined.

\section{Research object}

A schematic drawing of a hammer drill is shown on Fig. 2. It is well known that this type of machine use a set of working tools with different shape, size and weight. As parts of the mechanical system, these working tools also affect the force of the impact. Therefore, the working tools, just like the operating modes, are subject to optimization in order to maximize the machine efficiency. The present study is focused only on the chisel.

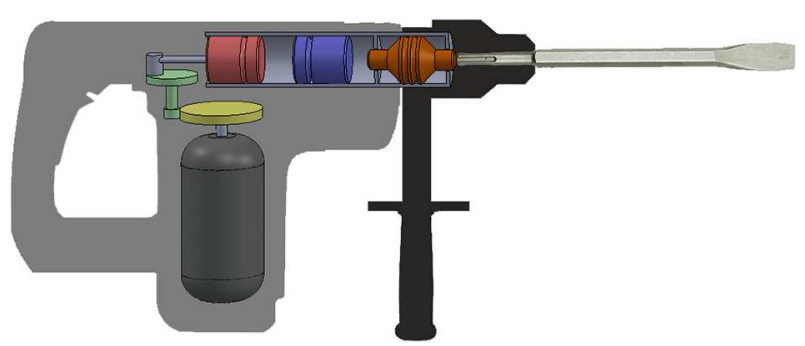

Fig. 2. Schematic drawing of a hammer drill

A suitable dynamic model has to be developed in order to give a numerical representation of the law for amending the force of impact. All parameters involved should be set down with their real values, preferably determined by an experiment.

After being created, the model should be validated with experimental data from the actual working process of the machine. The present study is the first step to the creation of such a model. It is aimed at determining the coefficients $\alpha$ and $\beta$ of the working tool of the hammer drill. 


\section{Dynamic model}

Drilling machines whose tool performs complex motion including rotation are examined in (Khulief et al., 2005; Yigit et al., 1998; Zare et al., 2011). In our case, the chisel moves only in the axial direction and it is assumed that the oscillations along this axis will be the most significant. Bending and twisting vibrations due to additional factors at this stage are neglected. Figure 3 shows the studied mechanical system which consists of a chisel and support. The latter is a pair of metal jaws clenching the chisel.
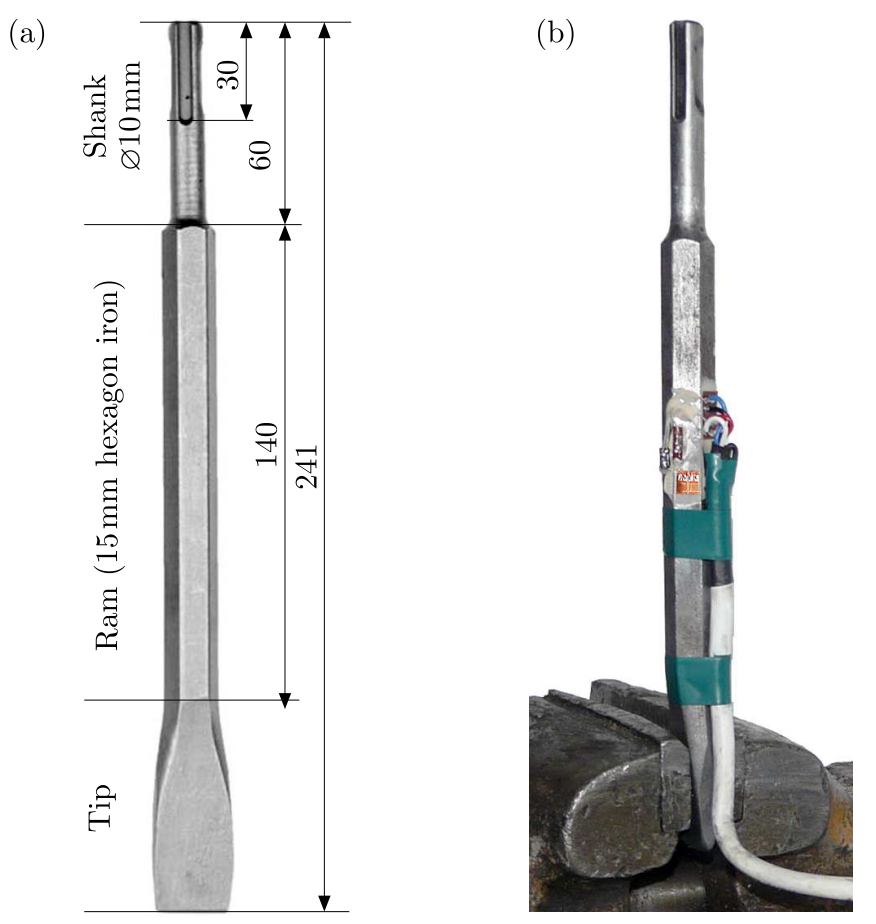

Fig. 3. Mechanical system: (a) sections and dimensions of the chisel, (b) fixing of the chisel

Figure 4 shows the dynamic model of the mechanical system. For the purpose of the modelling, the system is presented as a rod, fixed at its lower end, oscillating in the axial direction.

The movement of each section of the rod with constant cross-section is described with the following partial differential equation

$$
\frac{E}{\rho} \frac{\partial^{2} u_{i}(x, t)}{\partial x^{2}}-\frac{\partial^{2} u_{i}(x, t)}{\partial t^{2}}=0
$$

where $E$ is the elasticity modulus, $\rho$ - density of the material, $u_{i}(x, t)$ - coordinate, describing oscillations of the $i$-cross section of the rod. Since the cross section of the chisel is variable, it is divided into four sections, as shown in Fig. 3a (the dimensions are given in $\mathrm{mm}$ ):

- upper part of the shank, with diameter $10 \mathrm{~mm}$ and cut out channels,

- lower part of the shank, cylindrical, with diameter $10 \mathrm{~mm}$,

- ram - hexagonal, with diameter of the inscribed circle $15 \mathrm{~mm}$,

- tip with a relatively complex shape and cross section, decreasing from the ram to the end.

The finite element method has been used for solving equation (3.1). The rod is represented by 15 finite elements, shown in Fig. 4. The tip is discretized with 3 finite elements and all the other areas - with 4 elements. The cross section of the upper part of the shank is reduced by $20 \%$ compared to its lower part, due to fixing channels. The lengths of the ram elements are chosen 
(a)

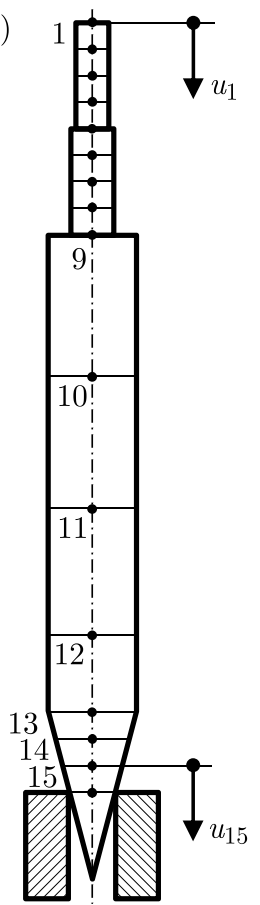

(b)

Element $i$

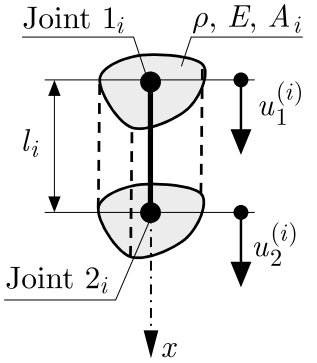

Fig. 4. Dynamic model with finite elements: (a) arrangement of the finite elements,

(b) single finite element

in such a way as to obtain a node at the place of bonding of the strain gauges, as shown in Fig. 3b. The elements of the tip are $10 \mathrm{~mm}$ long and thus its lower part (No. 15 in Fig. 4a) reaches the support. The cross section area of each element at the top is set according to the actual geometry.

All finite elements are of type shown in Fig. 4b (Reddy, 1984; Rao, 2004; Stelzmann et al., 2008). Each node has one degree of freedom, i.e. the finite element has two local degrees of freedom $\left(u_{1}^{(i)}\right.$ and $\left.u_{2}^{(i)}\right)$. The mass and stiffness matrices for the finite element used are as follows

$$
\mathbf{M}^{(e)}=\frac{\rho A_{i} l_{i}}{6}\left[\begin{array}{ll}
2 & 1 \\
1 & 2
\end{array}\right] \quad \mathbf{K}^{(e)}=\frac{E A_{i}}{l_{i}}\left[\begin{array}{cc}
1 & -1 \\
-1 & 1
\end{array}\right]
$$

It is more convenient to work with global coordinates for the formation of global matrices. The following connections between local and global coordinates should be taken into account during the transition to such global matrices

$$
u_{1}^{(1)}=u_{1} \quad u_{2}^{(1)}=u_{1}^{(2)}=u_{2} \quad \text { etc. }
$$

where $u^{(i)}(i=1,2, \ldots, 15)$ are the global coordinates (Reddy, 1984). Thus, equations (3.1) turn into a system of 15 ordinary differential equations of the second order, which take into account the boundary conditions.

The differential equation describing motion of the mechanical system is

$$
\mathbf{M} \ddot{\mathbf{q}}+\mathbf{C} \dot{\mathbf{q}}+\mathbf{K q}=\mathbf{0}
$$

where $\mathbf{M}$ is the mass matrix $(15 \times 15), \mathbf{K}$ is the stiffness matrix $(15 \times 15), \mathbf{C}=\alpha \mathbf{M}+\beta \mathbf{K}$ is the Rayleigh damping matrix, $\mathbf{q}=\left[u_{1}, u_{2}, \ldots, u_{15}\right]^{\mathrm{T}}$ is the coordinate vector, $\mathbf{0}$ is zero matrix $(15 \times 1)$.

The natural frequencies of the system are calculated in Matlab by solving equation (3.3) and without taking into account the internal resistance, i.e.

$$
\mathbf{M} \ddot{\mathbf{q}}+\mathbf{K q}=\mathbf{0}
$$


Equation (3.4) is brought into a normal appearance (Genov et al., 2007)

$$
\dot{\mathbf{X}}=\widetilde{\mathbf{A}} \mathbf{X}
$$

by introducing the following vector

$$
\mathbf{X}=[q, \dot{q}]^{\mathrm{T}}
$$

and the matrix $\widetilde{\mathbf{A}}$ is

$$
\widetilde{\mathbf{A}}=\left[\begin{array}{cc}
\mathbf{O}_{15 \times 15} & \mathbf{E}_{15 \times 15} \\
-\mathbf{M K} & -\mathbf{M O}_{15 \times 15}
\end{array}\right]
$$

In equation (3.7), $\mathbf{O}_{15 \times 15}$ is the zero matrix, $\mathbf{E}_{15 \times 15}$ is the identity matrix.

The natural frequencies of the system are obtained by determining the eigenvalues of the matrix $\widetilde{\mathbf{A}}$ by $Q-R$ algorithm - orthogonal projections (Watkins, 2004).

The first four values of the natural frequencies of the system thus obtained are given in Table 1.

Table 1. Natural frequencies

\begin{tabular}{|c|c|c|}
\hline $\begin{array}{c}\text { Natural } \\
\text { frequencies }\end{array}$ & $\begin{array}{c}f_{i} \\
{[\mathrm{~Hz}]}\end{array}$ & $\begin{array}{c}\omega_{i} \\
{[\mathrm{rad} / \mathrm{s}]}\end{array}$ \\
\hline \hline 1 & 5640 & 35419 \\
\hline 2 & 16920 & 106257 \\
\hline 3 & 26810 & 168366 \\
\hline 4 & 39530 & 248248 \\
\hline
\end{tabular}

\section{Influence of the internal resistance}

It has been assumed that the occurrence of the first two natural frequencies at free oscillations in the longitudinal direction is physically possible in the research model. The interval in Fig. 1 has been chosen to be from 35419 to $106257 \mathrm{rad} / \mathrm{s}$ (according to Table 1). According to equations (1.1), different values of the coefficients $\alpha$ and $\beta$ have been obtained for different values of the damping ratio $\xi$, as given in Table 2 .

Table 2. Values of $\alpha$ and $\beta$ depending on $\xi$

\begin{tabular}{|c|c|c|}
\hline$\xi$ & $\alpha$ & $\beta$ \\
\hline \hline 0.001 & 53.1288 & $1.41 \cdot 10^{-8}$ \\
\hline 0.010 & 531.288 & $1.41 \cdot 10^{-7}$ \\
\hline 0.100 & 5312.88 & $1.41 \cdot 10^{-6}$ \\
\hline
\end{tabular}

Differential equations (3.3) describing motion have been solved numerically by Matlab-Simulink, using the scheme in Fig. 5. For that purpose, the dimensions from Fig. 2 as well as the following values of the physical constants: $E=2.1 \cdot 10^{11} \mathrm{~Pa}$ and $\rho=7850 \mathrm{~kg} / \mathrm{m}^{3}$ have been taken.

The numerical solution of the problem using the values from Table 2, returned the results shown in Fig. 6 . The figure shows the relative deformation variation in time $\varepsilon=\left(u_{11}-u_{10}\right) / l_{10}$. Figure 6 shows that for the three values of $\xi$, the movement differs considerably (the time of damping is different), i.e. for successful modelling, it is of particular importance to set the exact value of $\xi$. 


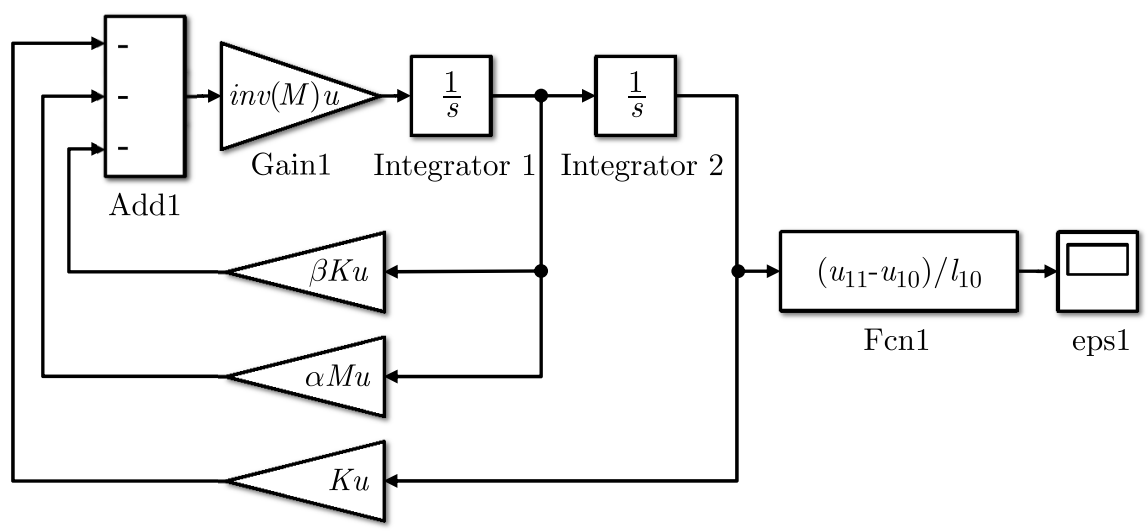

Fig. 5. Scheme for solving the differential equations in Matlab-Simulink

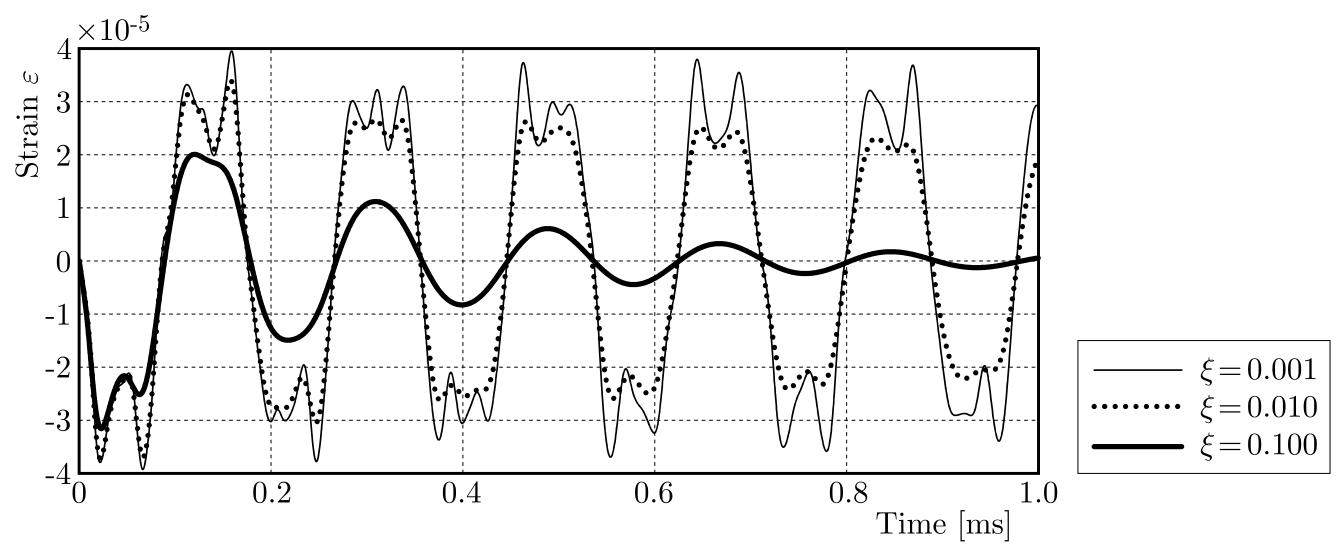

Fig. 6. Damped oscillations for different values of $\xi$

\section{Experimental determination of the chisel internal resistance by the method of damping oscillations}

One of the future goals of this project will be to measure the impact force of the machine under real operating conditions. Therefore, a decision has been taken to make tensometrical (strain) measurements. Free damped oscillations of the chisel arising from shock impact, have been thus registered in the course of the present study.

Figure 7 shows a scheme of the experimental installation. Strain gauges have been glued at two opposite points in the middle of the ram (Fig. 3b). Two equal half bridge T-rosettes have been used, each with two serially connected strain gauges, one of them in the longitudinal and the other - in the transverse direction to the chisel axis. The rosettes are connected in a full bridge circuit. The oscillation frequencies of the studied object are high, which makes the standard tensometric equipment inapplicable for strain registration. It has been, therefore, decided to use a high-frequency universal system for data acquisition (DAQ system) - National Instruments NI USB-6211 $(250 \mathrm{kS} / \mathrm{s}, 16 \mathrm{bit})$. Excitation voltage is applied to the bridge circuit with a suitable $12 \mathrm{~V} \mathrm{Li}$-ion rechargeable battery. The incoming information from the DAQ system is processed and recorded using software package LabVIEW ${ }^{\circledR}$. Calibration with reference weights has been made before the experiment. The chisel has been consecutively loaded with increasing weights of pure pressure in order to check the linearity and establish the conversion factors stress/strain.

In Fig. 7, the T-rosettes are not in their actual positions.

Figure 8 shows the recordings of damped oscillations after the impact on the studied system. Three recordings have been made with random forces of the impact, designated as FDO1, FDO2 


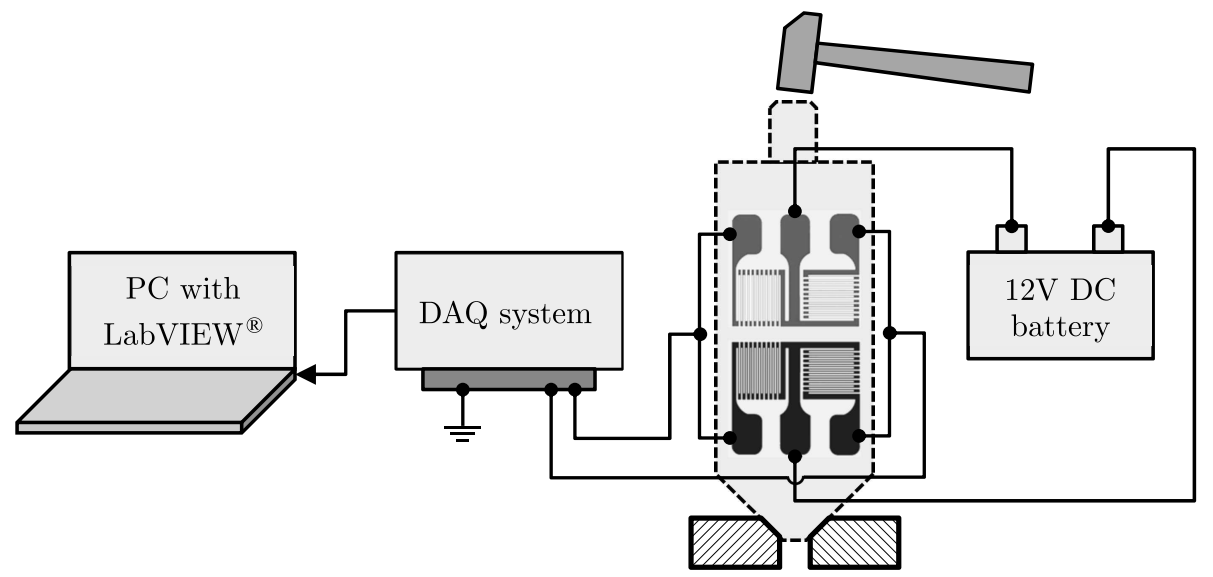

Fig. 7. Experimental installation scheme for registering strain upon impact

(a)

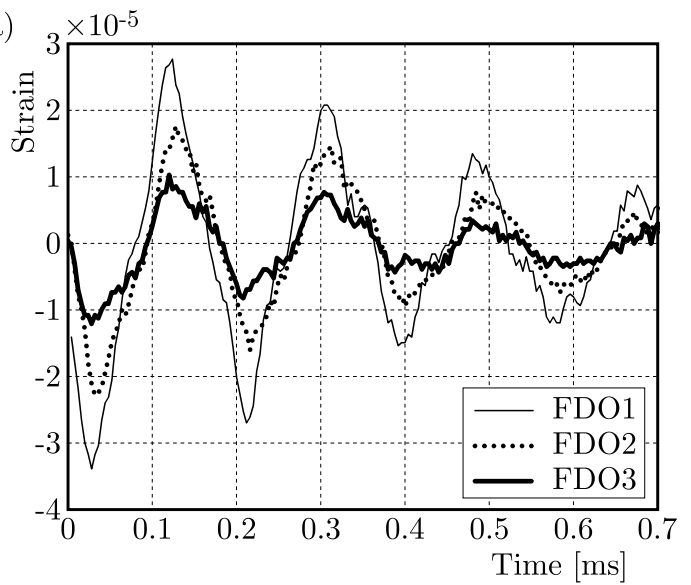

(b)

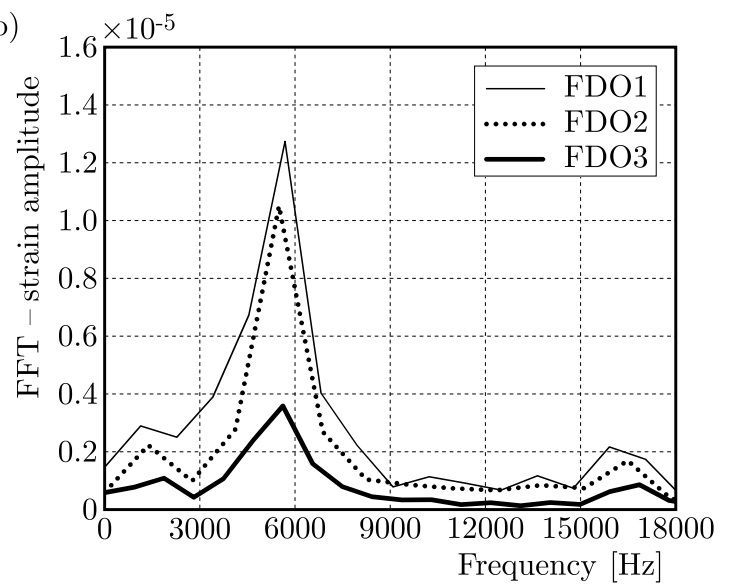

Fig. 8. Measurement results and frequency analysis

and FDO3 (FDO - free damping oscillations). On the left, the relative strain $\varepsilon$ is shown as a function of time and on the right - frequency analysis determining the damped oscillations frequency. Fast Fourier Transform (FFT) algorithm in Matlab is used for frequency analysis. The values obtained for the first and second frequency of the damped oscillations are given in Table 3. The results from the processing of all three recordings are similar.

Table 3. Frequencies of the damped oscillations

\begin{tabular}{|c|c|c|}
\hline Recording & $\begin{array}{c}f_{e 1}^{*} \\
{[\mathrm{~Hz}]}\end{array}$ & $\begin{array}{c}f_{e 2}^{*} \\
{[\mathrm{~Hz}]}\end{array}$ \\
\hline \hline FDO1 & 5682 & 15882 \\
\hline FDO2 & 5495 & 16483 \\
\hline FDO3 & 5618 & 16854 \\
\hline
\end{tabular}

Let us consider the first recording from Fig. 8 - FDO1. Damped oscillations have been observed with frequencies $\left(f_{e 1}^{*}=5682 \mathrm{~Hz}\right.$ and $\left.f_{e 2}^{*}=15882 \mathrm{~Hz}\right)$ close to the first two natural frequencies of the mechanical system (Table 1). It is assumed that the specific resistance coefficient $\xi$ of the damped oscillations is the same for the first and second frequency of the damped oscillations found from the experiment $\left(\omega_{e 1}^{*}=2 \pi f_{e 1}^{*}=35683 \mathrm{rad} / \mathrm{s}\right.$ and $\left.\omega_{e 2}^{*}=2 \pi f_{e 2}^{*}=99739 \mathrm{rad} / \mathrm{s}\right)$. Then its value can be determined on the basis of the lower frequency $\omega_{e 1}^{*}$. 
The differential equation, describing the damped oscillations with frequency $\omega_{e 1}^{*}$, is

$$
\ddot{u}+2 \xi \omega_{e 1} \dot{u}+\omega_{e 1}^{2} u=0
$$

The relationship between $\xi$ and $\omega_{e 1}$ is given by the equation

$$
\omega_{e 1}=\frac{\omega_{e 1}^{*}}{\sqrt{1-\xi^{2}}}
$$

where $\omega_{e 1}^{*}$ is the first frequency of the damped oscillations, determined by Fig. 8. Figure 9 shows four consecutive oscillations from the record, given in Fig. 8.

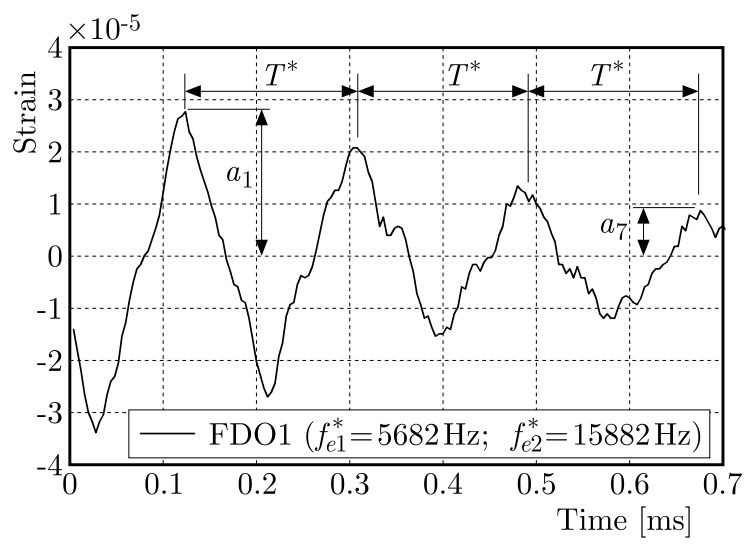

Fig. 9. Determining the resistance of damped oscillations

From Fig. 9 it can be determined that the ratio of the amplitudes $a_{1}$ and $a_{7}$ is 3.2. The following relation is valid for the amplitudes $i$ and $i+2 k$ ( $i$ and $k$ being positive integers)

$$
\frac{a_{i}}{a_{i+2 k}}=\exp \left(k \xi \omega_{e 1} \tau^{*}\right)
$$

Equation (5.3) can be resolved in relation to $\xi$, assuming that: $a_{1} / a_{7}=3.2, i=1, k=3, \omega_{e 1}^{*}$ is expressed by (5.2), and the period $\tau^{*}=2 \pi / \omega_{e 1}^{*}$. The result is $\xi=0.06$. Replacing $\xi$ with 0.06 , $\omega_{e 1}^{*}$ with $35683 \mathrm{rad} / \mathrm{s}$ and $\omega_{e 2}^{*}$ with $99739 \mathrm{rad} / \mathrm{s}$ in (1.1), we obtain the Rayleigh law coefficients

$$
\alpha=3153.7 \quad \beta=8.86 \cdot 10^{-7}
$$

Setting the values from equations (5.4) in numerical model (3.3) and applying the $Q-R$ algorithm - orthogonal projections (Watkins, 2004), we obtain the damped oscillation frequencies shown in Table 4.

Table 4. Frequencies of the damped oscillations

\begin{tabular}{|c|c|c|}
\hline $\begin{array}{c}\text { Natural } \\
\text { frequencies }\end{array}$ & $\begin{array}{c}f_{i}^{*} \\
{[\mathrm{~Hz}]}\end{array}$ & $\begin{array}{c}\omega_{i}^{*} \\
{[\mathrm{rad} / \mathrm{s}]}\end{array}$ \\
\hline \hline 1 & 5616 & 35270 \\
\hline 2 & 16837 & 105737 \\
\hline 3 & 26630 & 167238 \\
\hline 4 & 39135 & 245769 \\
\hline
\end{tabular}

The solution to differential equation (2.3) with these coefficients is shown in Fig. 10. 


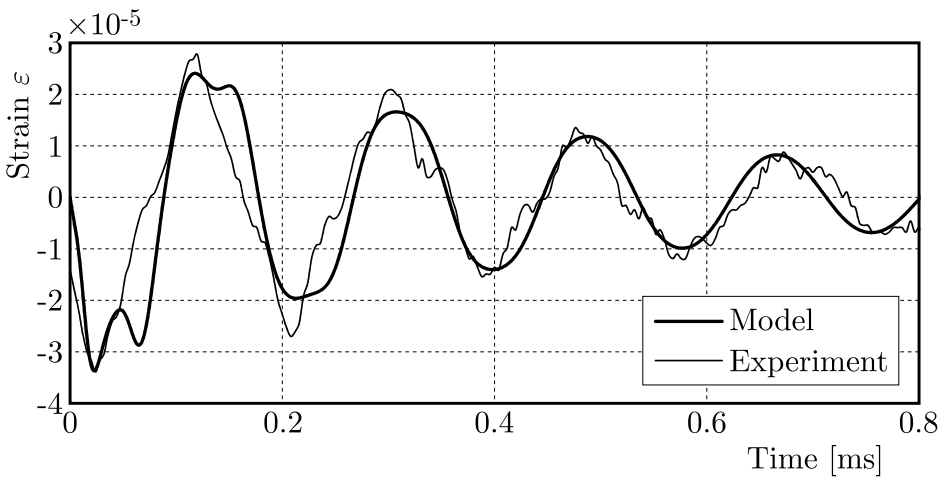

Fig. 10. Simulated and experimentally determined damped oscillations

\section{Summary}

Free damped oscillations of the chisel represented as a distributed parameters system have been studied in the present work. The dynamic model of the chisel was discretized with FEM and the problem was solved numerically with Matlab. The values of the coefficients $\alpha$ and $\beta$ (5.4), forming the Rayleigh damping matrix, have been experimentally determined in the given frequency range, after which they have been set in the numerical model. A comparison of the modelled oscillations with a real record has been made - see Fig. 10. The resulting close match shows that the proposed numerical model is acceptable and that the constants set in it have been correctly determined.

\section{References}

1. AdHIKari S., 2001, Damping models for structural vibration, Doctoral dissertation, University of Cambridge, 26-35

2. Alipour A., Zareian F., 2008, Study Rayleigh damping in structures; unceratinties and treatments, Proceedings of 14th World Conference on Earthquake Engineering, Beijing, China

3. Chopra A.K., 1995, Dynamics of Structures: Theory and Applications to Earthquake Engineering, New Jersey: Prentice Hall, 415

4. Chowdhury I., Dasgupta S.P., 2003, Computation of Rayleigh damping coefficients for large systems, Electronic Journal of Geotechnical Engineering, 8, Bundle C

5. Craig R.R., Kurdila A.J., 2006, Fundamentals of Structural Dynamics, John Wiley \& Sons, $501-504$

6. Dresig H., Holzweissig F., 2006, Machine Dynamics (in German), Springer-Verlag, 52, 417-427

7. Genov J., Polihronov G., Kralov I., 2007, Vibration of Vehicles, TU-Sofia, 25

8. Khulief Y.A., Al-Naser H., 2005, Finite element dynamic analysis of drillstrings, Finite Elements in Analysis and Design, 41, 13, 1270-1288

9. Man L., Corman D.G., 1995, Formulation of Rayleigh damping and its extensions, Computers and Mirrors, 57, 2, 277-285

10. Mevada H., Patel D., 2016, Experimental determination of structural damping of different materials, Procedia Engineering, 144, 110-115

11. RaO S.S., 2004, The Finite Element Method in Engineering, Butterworth-Heinemann, 430-431

12. Reddy J.N., 1984, An Introduction to the Finite Element Method, New York: McGraw-Hill, 123-131 
13. Sangeetha N., Sathish Kumar P., 2014, A study of vibration analysis for drill string using finite element analysis, IOSR Journal of Mechanical and Civil Engineering, 8, 60-74

14. Stelzmann U., Groth C., Müller G., 2008, FEM for Practitioners Volume 2: Structural Dynamics (in German), Auflage 5, Expert-Verlag, 89-91

15. Stevenson J.D., 1980, Structural damping values as a function of dynamic response stress and deformation levels, Nuclear Engineering and Design, 60, 2, 211-237

16. Watkins D.S., 2004, Fundamentals of Matrix Computations, John Wiley \& Sons, 356-371

17. Yigit A.S., Christoforou A.P., 1998, Coupled torsional and bending vibrations of drillstrings subject to impact with friction, Journal of Sound and Vibration, 215, 1, 167-181

18. Zare J., Hashemi S.J., RAshed G., 2011, Finite element analysis of drillstring lateral vibration, Scientific Research and Essays, 6, 13, 2682-2694

Manuscript received May 29, 2017; accepted for print August 30, 2017 